\title{
SYNTHESIS AND CHARACTERIZATION OF AMINO SILICONE OIL
}

\author{
Weilong Yang, Jing Tian, Zhenyu Wang, Chuncai Yang* \\ Jilin Institute of Chemical Technology Chengde Street, Jilin, China. \\ *Corresponding Author Email: 2941883707@qq.com
}

This is an open access article distributed under the Creative Commons Attribution License, which permits unrestricted use, distribution, and reproduction in any medium, provided the original work is properly cited

\section{ARTICLE DETAILS}

\section{Article History:}

Received 26 June 2018

Accepted 2 july 2018

Available online 1 August 2018

\section{ABSTRACT}

This paper deals with synthesis and characterization of amino silicone oil. The synthesis of amino silicone oil was made by Hexamethyldisiloxane (MM) octamethycyclotetrasiloxane (D4) and N, $\beta$-aminoethy- $\gamma$-aminopropylmethy dimethoxysilane (KH-602) through ring-opening polymerization. Fourier transform infrared spectroscopy (FT-IR) was used for the structure characterization. Its viscosity was measured by a rotary viscometer. Volatile matter content was measured by oven. Through the analysis of viscosity and yield, the optimal dosage of MM, KH-602 and Tetramethylammonium hydroxide was obtained. The optimal reaction conditions were obtained by different amounts of MM, KH-602 and Tetramethylammonium hydroxide. Results showed that amino silicone can be obtained by this method. Amino silicone oil has good performance as a carbon fiber oil agent.

\section{KEYWORDS}

Amino silicone oil, ring-opening polymerization.

\section{INTRODUCTION}

Organosilicon compounds have been widely used in various industries because of their lubricity, flexibility, non - toxicity, no environmental pollution and low cost [1-3]. In the spinning process of PAN-based carbon fiber precursor, oil is applied before and after the drying densification process, which can prevent adhesion or doubling between single fiber filaments. Because when the adhered fibers are peeled off, the surfaces of the carbon fibers are scratched, new defects are generated, the quality of the protofilaments is reduced, and the performance is lowered.

In the pre-oxidation process of precursor, the oil agent on the surface of precursor can prevent the surface of precursor from generating new defects. Preoxidation process is an exothermic reaction process, due to the accumulation of heat can lead to local overheating, which resulting in thermal adhesion between filaments or filament, also if you want to open the thermal adhesion or filament, it will form new defects on the surface of the fiber. At this time, the multifunctional oil agent can prevent the occurrence of hot adhesion or hot drawing phenomenon, and prevent the occurrence of new defects.

In the low-temperature carbonization process, $30 \sim 40 \%$ of the fiber filament's weight is discharged in the form of volatile gas or tar, especially in the temperature range of $320 \sim 650^{\circ} \mathrm{C}$, so that a large amount of byproducts can also generate thermal adhesion and hot melt and filament merging between monofilaments, which resulting in defects on the surface of PAN-based carbon fiber filaments [4]. In the low-temperature carbonization zone, the multifunctional oil agent continues to play its protective role to prevent defects on the fiber surface.

The multifunctional oil agent forms a protective film on the surface of the precursor to prevent the friction damage of the precursor during spinning, preoxidation and carbonization. When the PAN-based carbon fiber precursor with a single diameter of about $10 \sim 13 \mu \mathrm{m}$ is contacted with the active rod and passive coil, the friction will cause damage to the surface of PAN-based carbon fiber precursor. The oil agent can play a role of lubricant, and the film formed on the surface of the single fiber yarn can protect the surface of the fiber yarn from being damaged and smoothly pass through spinning [5].
Amino silicone oil was known for its large adsorption capacity, good filmforming property, strong bond with fiber and its ability to impart soft and smooth hand feel to fabrics. This paper deals with synthesis and characterization of amino silicone oil [6].

\section{EXPERIMENTAL}

\subsection{Materials}

Octamethycyclotetrasiloxane (D4), (Shouguang Lisheng Chemical); N, $\beta$ aminoethy- $\gamma$-aminopropylmethy dimethoxysilane (KH-602), (Jiangsu Chenguang Chemical); Hexamethyldisiloxane (MM), (Adamas reagent); Tetramethylammonium hydroxide (TMAH), (Tianjin Guangfu Chemical)

\subsection{Synthesis of Amino Silicone oil}

The synthesis of amino silicone oil was carried out in four steps. Hexamethyldisiloxane, octamethycyclotetrasiloxane, N, $\beta$-aminoethy- $\gamma$ aminopropylmethy dimethoxysilane, tetramethylammonium hydroxide solution and a certain amount of deionized water were added into the reaction flask at one time, and the temperature was raised to $80 \sim 90^{\circ} \mathrm{C}$, and the reaction was carried out of 1 hour under the condition of introducing nitrogen. Thereafter, the temperature was raised to $100 \sim 110^{\circ} \mathrm{C}$ to react for $4 \sim 6$ hours. After the reaction was completed, the temperature was raised to $145 \sim 150^{\circ} \mathrm{C}$ for 1 hour to decompose the catalyst. Obtaining amino silicone oil by a method of vacuumizing to remove low-boiling substances under the same temperature.

\subsection{Emulsion Characterization}

The FT-IR spectra were recorded by the Shimadzu Japan instrument with $\mathrm{KBr}$ pellets. The background spectrum of the $\mathrm{KBr}$ pellet was subtracted from the sample spectrum. The viscosity of amino silicone oil was analyzed by rotary viscosimeter (Brookefield,USA). Quasi - weighed 5g samples were placed in an oven which made by Shanghai huqin instrument equipment co., Itd. at $150{ }^{\circ} \mathrm{C}$ for 3 hours, and the weight loss was measured 


\section{RESULTS AND DISCUSSION}

\subsection{FT-IR spectra}

Figure 1 shows the FT-IR characterization of synthetic animo silicone oil.It can be seen from the spectrum that the telescopic vibration peak $\mathrm{C}-\mathrm{H}$ in $\mathrm{CH} 3$ at $2963 \mathrm{~cm}-1$ and $2905 \mathrm{~cm}-1$, the in-plane bending vibration peak C$\mathrm{H}$ at $1413 \mathrm{~cm}-1$, the deformation vibration peak and the plane rocking vibration peak $\mathrm{C}-\mathrm{H}$ in $\mathrm{Si}-\mathrm{CH} 3$ and the telescopic vibration peak $\mathrm{Si}-\mathrm{C}$ at $1261 \mathrm{~cm}-1,864 \mathrm{~cm}-1$ and $800 \mathrm{~cm}-1$, the telescopic vibration peak Si-O-Si at $1093 \mathrm{~cm}-1$ and $1021 \mathrm{~cm}-1$, the bending vibration peak $-\mathrm{NH} 2$ and $-\mathrm{NH}$ at $1593 \mathrm{~cm}-1$, the rocking vibration peak -NH2 at $702 \mathrm{~cm}-1$.From the above peaks, we can preliminarily judge that the synthesized substance is the expected amino silicone oil.

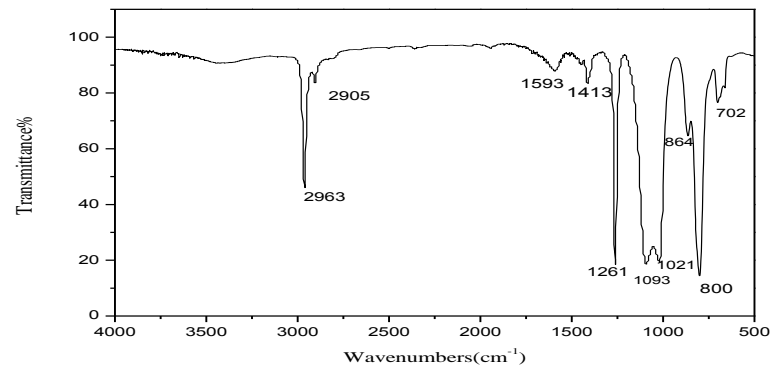

Figure 1: FTIR spectra of amino silicone oil

\subsection{Effect of MM dosage on technical indicators of amino silicone oil products}

Table 1: Relationship Between Viscosity of Amino Silicone Oil and Dosage of MM

\begin{tabular}{|c|c|c|}
\hline $\begin{array}{l}\text { Dosage of } \\
\text { MM\% }\end{array}$ & Viscosity(cp) & Yield\% \\
\hline 0.7 & 840 & 75.2 \\
\hline 1.4 & 780 & 75.2 \\
\hline 2.9 & 500 & 75.3 \\
\hline
\end{tabular}

Notes. The mass fractions of $\mathrm{KH}-602$ and $\left(\mathrm{CH}_{3}\right)_{4} \mathrm{NOH}$ were $40 \%$ and $0.17 \%$.

Table 1 shows that with the increase of MM dosage, the viscosity of amino silicone oil gradually decreases, and the yield of amino silicone oil has little influence. In addition, because water can also be used as a blocking agent to reduce viscosity, if water is not removed in time in the reaction, the molar mass of amino silicone oil will be reduced. The dosage of MM used in this experiment is $1.4 \%$.

\subsection{Influence of KH-602 dosage on properties of amino silicone oil}

Table 2: Influence of KH-602 Dosage on Technical Indicators of Amino Silicone Oil

\begin{tabular}{|lll|}
\hline $\begin{array}{l}\text { Dosage of } \\
\text { KH-602\% }\end{array}$ & Viscosity(cp) & Yield\% \\
\hline 15 & 1040 & 81.1 \\
30 & 800 & 80.2 \\
40 & 780 & 75.2 \\
\hline
\end{tabular}

Notes. The mass fractions of MM and $\left(\mathrm{CH}_{3}\right)_{4} \mathrm{NOH}$ were $1.4 \%$ and $0.17 \%$.
Table 2 shows that with the increase of KH-602 dosage, the viscosity and yield of amino silicone oil decreased. It shows that the ammonia value of amino silicone oil is mainly determined by the ratio between monomers. As the amount of KH-602 increases and the amount of deionized water used in the system increases proportionally, the catalytic activity of the catalyst decreases, resulting in the decrease of product viscosity and yield.

\subsection{Influence of Catalyst dosage on properties of amino silicone oil}

Basic metal oxides and quaternary ammonium hydroxides can be used as catalysts for the synthesis of amino silicone oils, and the catalytic activity of quaternary ammonium hydroxides is higher. When TMAH is used as catalyst, the influence of catalyst dosage on viscosity and yield of amino silicone oil is shown in the table 3 .

Table 3: Influence of Catalyst Dosage on Technical Indicators of Amino Silicone Oil

\begin{tabular}{|lll|}
\hline $\begin{array}{l}\text { Dosage of } \\
\text { catalyst } \%\end{array}$ & Viscosity $(\mathrm{cp})$ & Yield\% \\
\hline 0.08 & 1080 & 80.2 \\
0.17 & 1040 & 81.1 \\
0.35 & 930 & 81.2 \\
\hline
\end{tabular}

Notes. The mass fractions of MM and KH-602 were $1.4 \%$ and $15 \%$.

Table 3 shows that when the amount of catalyst is greater than $0.08 \%$, the polymerization time of $4 \sim 6$ hours can basically reach equilibrium, and the viscosity of amino silicone oil is stable and the yield is stable. Considering the economic value, it is better to use $0.17 \%$ catalyst.

\section{CONCLUSION}

The best reaction conditions for the synthesis of amino-modified silicone oil can be known from this document. Amino silicone oil synthesized in this paper had good stability and was colorless and transparent. Its application in carbon fib will be reported in a separate article.

\section{REFERENCES}

[1] Du, Z. 1990. Amin organosilicon chemistry. Beijing: Higher Education Press. ISBN:7040022192

[2] Huang, W. 1998. Amino modified silicone oil and fabric softener. Journal of Organosilicon materials and applications, (5), 16-18.

[3] Sun, S., Li, J. 2007. Development and application of amino silicone oil softener. Journal of Textile guide, 25 (5), 34-37.

[4] Msarah, M., Alsier, A. 2018. Protein Digestibility and Amino Acid Content of Malaysian Local Egg Pro tein Prepared by Different Methods. Environment and Ecosystem Science, 2 (1), 07-09.

[5] Sun, X. 2012. Analyzing and application of oiling agent to Polyacylonitrile (PAN)-based carbon fiber precursor. Changchun: Liangui Li.

[6] Zhang, H., Liu, L., Liu, J. 2009. Synthesis and applications of amino silicone oil. Journal of Westleather, (23), 29-32. 